\title{
Propagation of ultrashort pulses in single-mode fiber
}

\author{
V. A. Astapenko, E. S. Manuylovich, V. A. Bagan
}

V. A. Astapenko, E. S. Manuylovich, V. A. Bagan, "Propagation of ultrashort pulses in single-mode fiber," Proc. SPIE 9450, Photonics, Devices, and Systems VI, 945016 (6 January 2015); doi: 10.1117/12.2070395

SPIE. Event: Photonics Prague 2014, 2014, Prague, Czech Republic 


\title{
Propagation of ultrashort pulses in single-mode fiber
}

\author{
V.A.Astapenko, E.S.Manuylovich, V.A. Bagan
}

Moscow Institute of Physics and Technology, 141700 Russia, Dolgoprudny, Institutsky side street 9

\begin{abstract}
Propagation of ultrashort pulses in dispersing media and optical fibers is calculated and analyzed. An analytical expression for the electric field of ultra short pulse after propagating certain distance $\mathrm{z}$ is derived in the second order dispersion theory. The propagation of a Gaussian pulse is considered.
\end{abstract}

ultra-short pulses, optical fiber, dispersion

\section{Introduction}

Laser systems that generate femtosecond pulses of near-IR, visible and UV range are widely used in experimental physics and technological applications from the end of the last century. Their application includes creating ultrafast semiconductor switching devices as well as submicron processing techniques and modifications of various materials.

The analysis of propagation of ultrashort electromagnetic pulses in optical waveguides is of great interest for future tasks such as fast data transfer and femtosecond near-field microscopy. The sufficient gain bandwidth as well as synchronization of the greatest possible number of longitudinal modes of the laser cavity are important problems when obtaining femtosecond laser pulses. These problems require special actions to compensate the dispersion in all intracavity elements. In this paper we shall not mention the problem of generation of ultrashort pulses (for single-cycle pulse generation in fiber see [1]), but we will consider the features of their propagation in the optical fiber.

The purpose of this work is to numerically simulate the propagation of ultrashort pulses in optical fiber and determine the maximum transfer distance depending on pulse parameters.

\section{Propagation of ultrashort pulses in the dielectric medium}

Main characteristics of ultrashort electromagnetic pulses and generation methods are well covered in the literature [2-4]. In this section we describe the general regularities of the spreading of an ultrashort pulse in a dispersive medium [5].

The wave equation for the electric field propagating along the axis $\mathrm{z}$ is:

$$
\frac{\partial^{2} \mathbf{E}}{\partial z^{2}}-\frac{1}{c^{2}} \frac{\partial^{2} \mathbf{D}}{\partial t^{2}}=0
$$

where

$$
\mathbf{D}(z, t)=\int_{0}^{\infty} \varepsilon(\tau) \mathbf{E}(z, t-\tau) d \tau
$$

is the electric displacement field in a homogeneous isotropic nonmagnetic medium without spatial dispersion. We perform a Fourier transform

$$
E(t, z)=\int_{-\infty}^{+\infty} E(\omega) e^{i(\omega t-k z)} d \omega
$$

then from (1) and (2) we obtain the dispersion relation

Photonics, Devices, and Systems VI, edited by Pavel Tománek, Dagmar Senderáková, Petr Páta, Proc. of SPIE Vol. 9450, 945016 - (c) 2015 SPIE · CCC code: 0277-786X/15/\$18 · doi: 10.1117/12.2070395 


$$
k(\omega)=\frac{\omega}{c} \sqrt{\varepsilon(\omega)}=\frac{\omega}{c} n(\omega)
$$

\section{Propagation of ultrashort pulses in a single-mode waveguide}

There is the fundamental longitudinal mode $\mathrm{HE}_{11}$ in dielectric waveguides. For this mode there is no the cutoff frequency. We consider a weakly guiding fibers with a stepwise refractive index profile and $n_{1} \approx n_{2}$. Optical fiber is single-mode (for transverse modes) when:

$$
V \leq 2.405
$$

where

$$
V=\frac{2 \pi a}{\lambda} \sqrt{n_{1}^{2}-n_{2}^{2}}
$$

is the dimensionless frequency (waveguide parameter).

The dispersion relation for $\mathrm{HE}_{11}$ mode is well known:

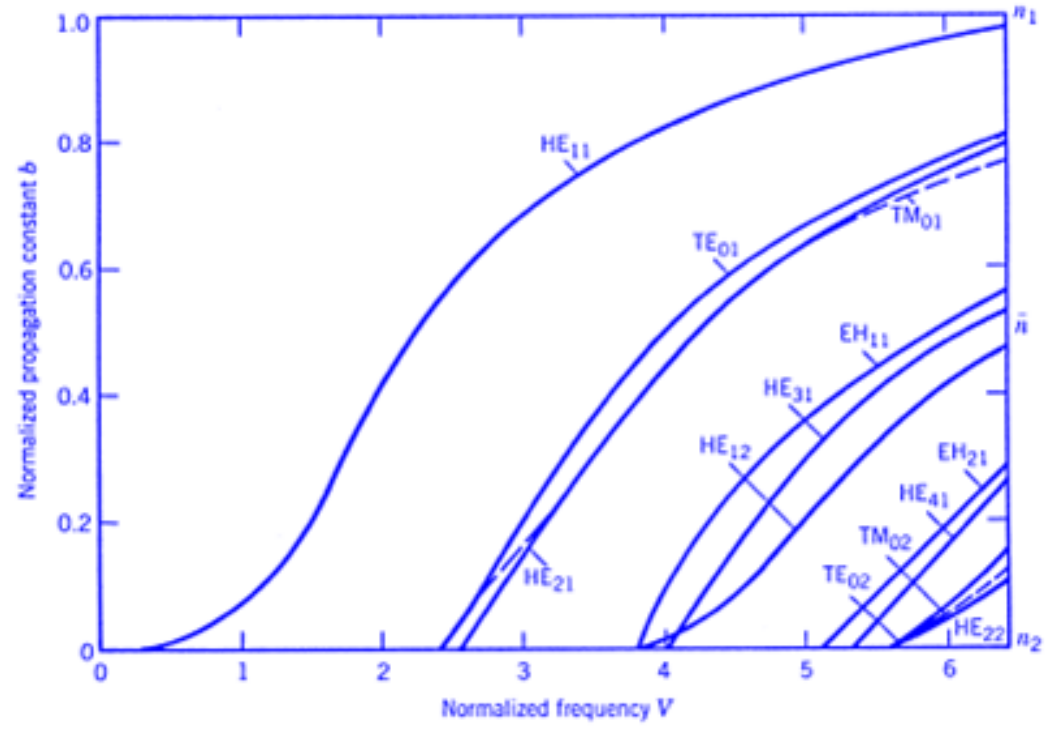

Fig.1 Dispersion relation of the lower modes of an optical fiber

The propagation constant of the electromagnetic field along the axis of the waveguide can be expressed through the effective refractive index:

$$
\beta(V)=\frac{2 \pi}{\lambda}\left(n_{2}+n_{1} b(V) \Delta\right)
$$

where

$$
\Delta \cong \frac{n_{1}-n_{2}}{n_{1}}
$$

and $\lambda$ is the wavelength in vacuum.

Pulse propagation in a homogeneous cylindrical waveguide which is placed in vacuum, is considered in [6]. Pulse shape distortion due to dispersion spreading is calculated. New lasers based on chromium-forsterite crystals emit in the vicinity of wavelengths of $1250 \mathrm{~nm}$ that is close to the minimum dispersion of quartz. 
To calculate the dispersion pulse distortion in optical fiber we use (3) where we make the change of variables:

$$
k(\omega) \rightarrow \beta_{11}(\omega)
$$

where $\beta_{11}(\omega)$ is propagation constant of $\mathrm{HE}_{11}$ mode. Propagation constant is expressed by the formula (9) through the effective refractive index for this mode and the refractive indices of the core and cladding of the fiber.

We choose the shape of the spectrum of forsterite laser as Gaussian because of its luminescence band shape:

$$
E(\omega)=A \exp \left(-\left(\omega-\omega_{0}\right)^{2} \tau^{2} / 4\right) .
$$

Such a spectrum corresponds to the Morlet wavelet [7]

$$
E(t)=\frac{A}{\tau \sqrt{\pi}} e^{-i \omega_{0} t} e^{-t^{2} / \tau^{2}} .
$$

Fig. 2 presents a pulse electromagnetic field with a carrier wavelength $\lambda_{0}=1250 \mathrm{~nm}, \tau=10 \mathrm{fs}_{S}$, which is given by the real part of formula (13).

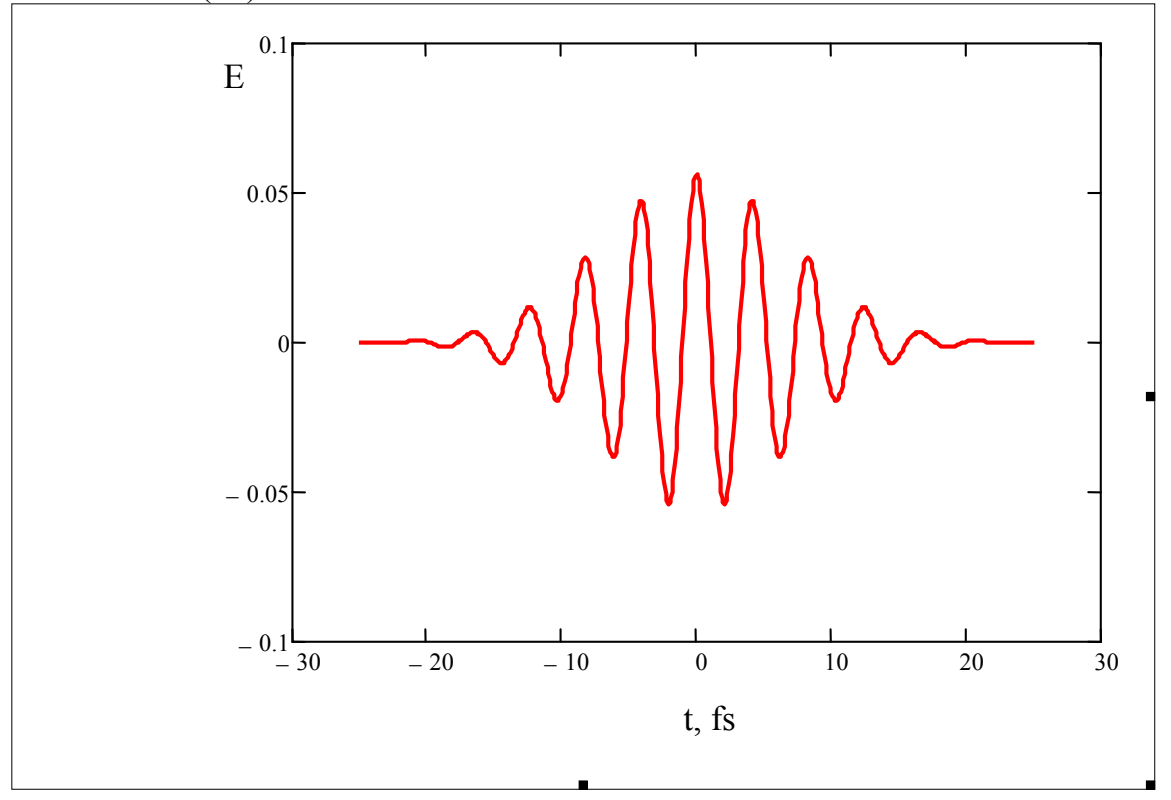

Fig.2 The light pulse (11) $\left(\lambda_{0}=1250 \mathrm{~nm}, \tau=10 \mathrm{fs}_{\mathrm{S}}\right)$ at the inlet of the fiber

Consider the dispersion spreading and delay of pulse (11) with parameters $\lambda_{0}=1250 \mathrm{~nm}, \tau=10 \mathrm{fS}_{\mathrm{S}}$ during its propagation in SMF28 fiber. This fiber has the following characteristics: core radius $a=4.1 \mu \mathrm{m}, \Delta=0.0036$. Note that in this case $V=2.104$, i.e. there is a single mode regime. Using formula (3) with the substitution (11) after separation of the pulse carrier frequency, we obtain

$$
\begin{gathered}
E(z, t)=\frac{A}{2 \pi} \int_{-\infty}^{\infty} \exp \left(i \omega \tau_{z}\right) \exp \left(\frac{-\left(\omega-\omega_{0}\right)^{2} \tau^{2}}{4}\right) \exp \left[i \omega z\left(n_{e f}\left(\omega_{0}\right)-n_{e f}(\omega)\right) / c-\alpha(\omega) z\right] d \omega \\
\tau_{z}=t-n_{e f}\left(\omega_{0}\right) z / c .
\end{gathered}
$$

where

$$
n_{e f}(\omega)=\frac{c \beta_{11}}{\omega}
$$


Value $\omega z\left(n_{e f}\left(\omega_{0}\right)-n_{e f}(\omega)\right) / c$ shows the magnitude of the phase shift due to the dispersion and $\alpha(\omega)$ is the attenuation coefficient. Variable $\tau_{z}$ is the delay that occurs during the propagation of a monochromatic wave with a frequency equal to the center frequency of the pulse. In our case the attenuation can be neglected.

Figure 3 shows the result of numerical calculation of pulse (11) propagation in SMF28 fiber. The calculation was performed by formulas (12) - (13).

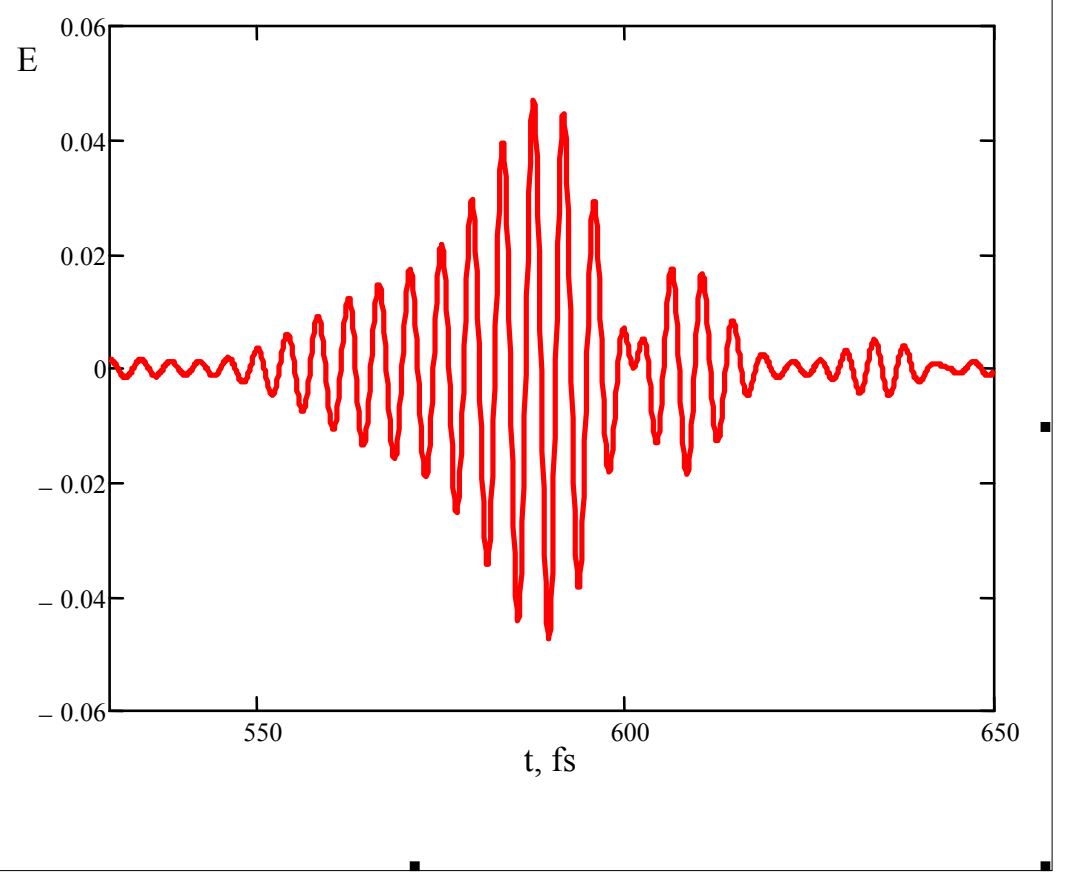

Fig. 3 Shape of the pulse shown in Fig. 2 after propagation in fiber SMF28 at distance $z=1 \mathrm{~cm}$

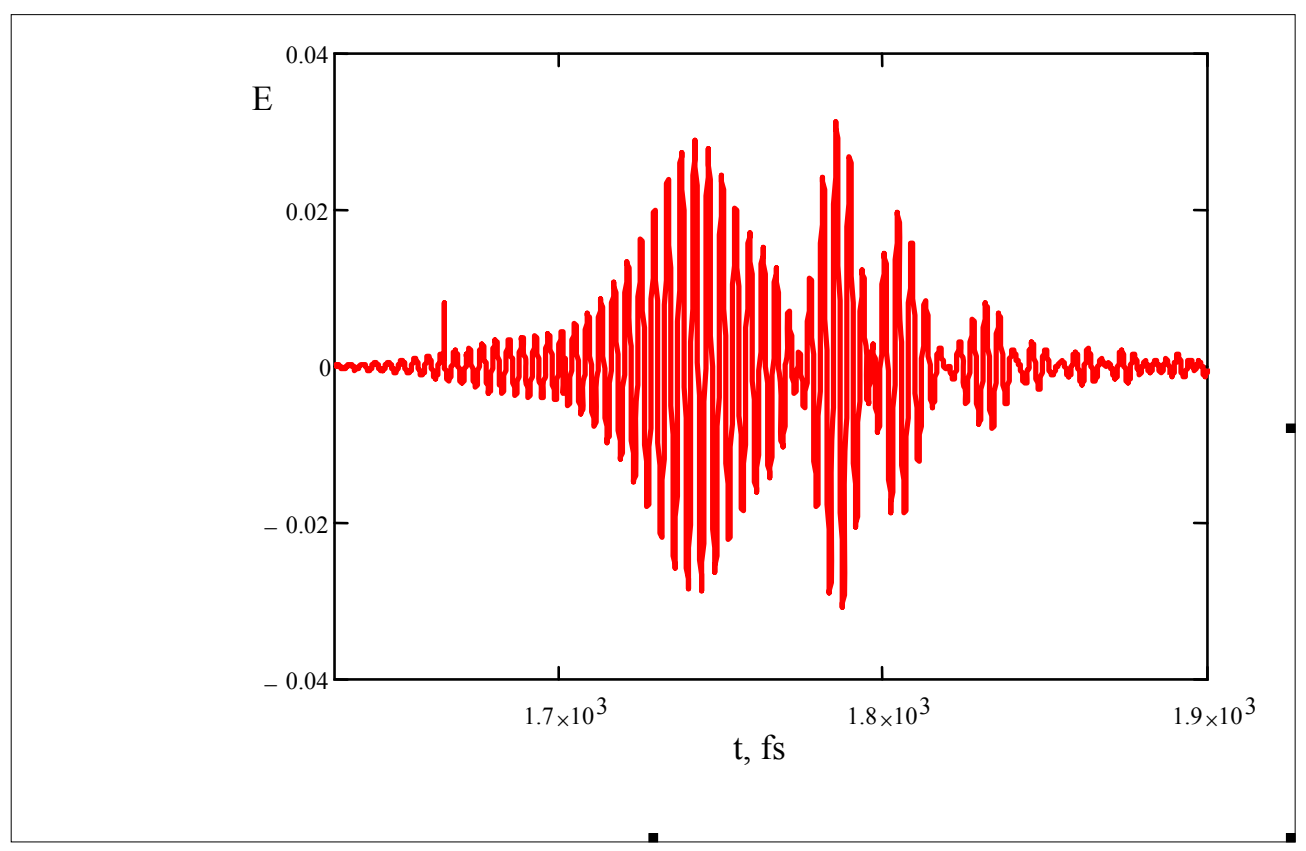

Fig. 4 Shape of the pulse shown in Fig. 2 after its propagation in fiber SMF28 at distance $z=3 \mathrm{~cm}$ 
The results of calculation of a short pulse propagation with a duration of $12 \mathrm{fs}$ ( $\tau=6 f s$ ) and carrier wavelength $\lambda_{0}=1064 \mathrm{~nm}$ (ytterbium laser) are shown in Figures 5 and 6. Optical fiber parameters: core radius a $=3.5$ $\mu \mathrm{m}, \Delta=0.003(V=1.928)$.

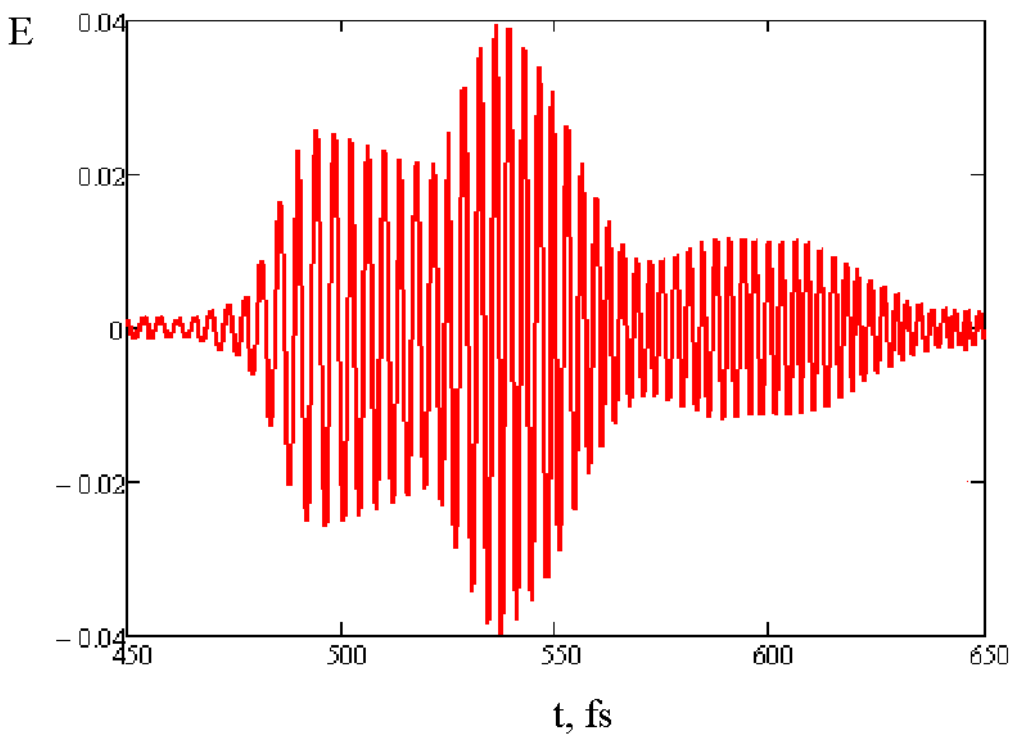

Fig. 5 Shape of the 12-fs pulse after its propagation at distance $z=1 \mathrm{~cm}$

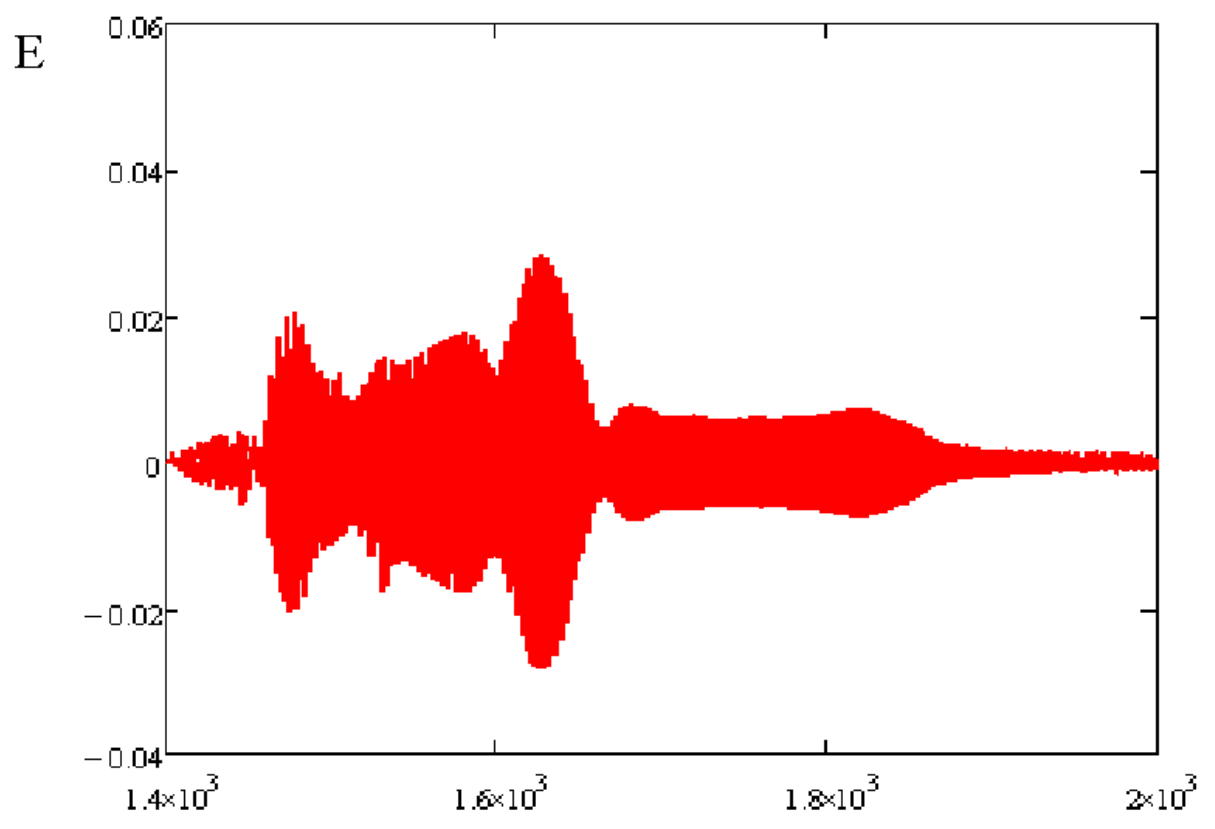

$\tau$, fs

Fig. 6 Shape of the 12-fs pulse after its propagation at distance $z=3 \mathrm{~cm}$

The data shown in Figures 3, 4 and 5, 6 demonstrate that the propagation of ultrashort pulses of $20 \mathrm{fs}$ and $12 \mathrm{fs}$, even for short distances $(1 \mathrm{~cm}$ and $3 \mathrm{~cm})$ significantly change the pulse shape, and the pulse duration increases. It is also clear that the pulse propagation velocity decreases by decreasing the group velocity in the optical fiber. Thus, short segments of optical fibers could be used for dispersion dilation of ultrashort pulses. The analysis shows that the absorption does not significantly impact on the pulse at such small distances.

Carrier frequency change has no significant effect on the propagation of ultrashort pulses in the fiber. 


\section{Acknowledgements}

The work has been done with the financial support of the RF Ministry of Education and Science within the framework of the Government order (research work No. 1940).

\section{References}

[1] Krauss G., Lohss S., Hanke T., Sell A., Eggert S., Huber R., Leitenstorfer A., "Synthesis of a single cycle of light with compact erbium-doped fibre technology", Nature Photonics, 33 - 36 (2010)

[2] Gets A.V., Krainov V.P.. "Ionization of atoms by attosecond pulses " Contribution to Plasma Physics. Vol. 53. pp. 18 (2013).

[3] Johnson P.B., Chirsty R.W., "Optical constants of the noble metals ", Physical Review B, Vol. 6., pp. 4370-4379 (1972).

[4] Morshnev S.K., [Electromagnetic waves in dielectric waveguides], MIPT, Moscow (2004).

[5] Moses J., Huang S.-W., Hong K.-H. et al., "Highly stable ultrabroadband mid-IR optical parametric chirped-pulse amplifier optimized for superfluorescence suppression ", Optical Letters, Vol. 34., pp. 1639-1641 (2009).

[6] Kundu M., Kaw P.K., Bauer D., "Laser-cluster interaction with subcycle pulses" Physical Review A, Vol. 85.,No. 2, pp. 023202-1-9 (2012).

[7] Dong L., Li J., McKay H.A., Fu L., Thomas B.K., "Large Effective Mode Area Optical Fibers for High Power Lasers", Proc. SPIE 7195 (2009). 